Short Research Communication

\title{
Mutual Regulation of FOXM1, NPM and ARF Proteins
}

\author{
Bulbul Pandit and Andrei L. Gartel ${ }^{凶}$ \\ Department of Medicine, University of Illinois at Chicago, Chicago, IL, 60612, USA \\ $\triangle$ Corresponding author: Andrei L. Gartel, University of Illinois at Chicago, Department of Medicine, 840 S. Wood St., Room 1041, Chicago, \\ IL 60612, USA; (312) 996-1855 (Telephone), (312) 413-0342 (Fax), agartel@uic.edu \\ (C) 2015 Ivyspring International Publisher. Reproduction is permitted for personal, noncommercial use, provided that the article is in whole, unmodified, and properly cited. \\ See http:/ /ivyspring.com/terms for terms and conditions.
}

Received: 2015.02.02; Accepted: 2015.03.16; Published: 2015.04.28

\begin{abstract}
ARF, NPM and FOXMI proteins interact with each other in mammalian cells. We showed previously that proteasome inhibitors suppress not only FOXMI expression, but also the expression of ARF and NPM proteins. Using RNA interference we found that the depletion of each of these proteins by RNAi in human cancer HeLa cells leads to down-regulation of the two other partners, suggesting that these proteins stabilize each other in human cancer cells. Since the suppression of FOXMI is one of hallmarks of proteasome inhibition, suppression of ARF and NPM by proteasome inhibitors may be explained in part as a secondary effect of downregulation of FOXM1 that modulate stability of ARF and NPMI proteins.
\end{abstract}

Key words: ARF, NPM, FOXM1, RNA interference

\section{Introduction}

Forkhead box M1, FOXM1, is a transcription factor of the Forkhead family, which is overexpressed in a large number of human cancer [1]. FOXM1 consists of a Forkhead-DNA binding and an acidic transactivation domain. FOXM1 regulates the expression of genes involved in the cell cycle progression and the proper execution of mitosis. The genes that are activated by FOXM1 initiate the G1/S and $\mathrm{G} 2 / \mathrm{M}$ transition and $\mathrm{S}$ and $\mathrm{M}$ phase execution. It was identified as one of the most commonly overexpressed proteins in solid tumors based on microarray data [2]. FOXM1 presents a proliferation specific pattern of protein expression. It is also shown to be involved in the metastatic and angiogenic progression of cancer. The expression of FOXM1 is increased by oncogenic proteins such as c-Myc, AKT, H-Ras,etc. Conversely, the expresion of FOXM1 is downregulated by tumor-suprressor such as PTEN, $\mathrm{Rb}$, p53, etc. As a result, it represents a bona-fide target for anticancer treatment [3].

Nucleophosphomin (NPM) 1 is a universally expressed chaperone phosphoprotein associated with the cell cycle regulation. It shuttles between the cytoplasm and the nucleus, however it mostly exists in the nucleolus [4]. The expression of NPM increases following mitogenic stimuli and it is involoved in proliferation and regulation of growth of many cancers. It can interact with a several cellular proteins in various parts of cells, such as p53, FOXM1, etc. affecting their stabilty and activity $[5,6]$.

ARF is a tumor suppressor protein transcribed from the alternate reading frame of the INK4a/ARF locus (CDKN2A). It is also known as p14 ${ }^{\mathrm{ARF}}$ in human and p19ARF in mouse. The expression of ARF leads to inhibition of aberrant cell cycle progression by direct inhibition of MDM2 (negative regulator of p53). ARF mediated inhibition of MDM2 leads to activation of the p53 transcription factor resulting in cell cycle arrest or apoptosis following DNA damage and or oncogenic activation. It is also been demonstrated that ARF can initiate tumor suppressive function independent of the p53 status. It resides in the nucleolus and has been shown to interact with NPM1 [7]. ARF is inactivated in many types of cancer and as a result the physiological expression level of ARF (p14 ${ }^{\mathrm{ARF}}$ ) is usually very low in tumor cells [8]. 
Here, we will provide evidence that all these three proteins stabilize each other in cancer cells and suppression of ARF and NPM1 could be partially explained as a result of FOXM1 initial suppression by proteasome inhibitors.

\section{Materials and Methods}

\section{Cell Culture}

HeLa (human cervical cancer cells) were purchased from ATCC. The cells were maintained in DMEM medium supplemented with $10 \%$ fetal bovine serum (Atlanta Biologicals) and $1 \%$ penicillin-streptomycin (GIBCO). All the cells were maintained at $37^{\circ} \mathrm{C}$ in $5 \% \mathrm{CO}_{2}$.

\section{Transfection Experiments}

HeLa cells were transiently transfected with a control (AACAGUC G CGUUUGCGACUGGUU) small interfering RNA (siRNA) and siRNA specific to FOXM1 (GGACCACUUUCCCUACUUUUU) or ARF (CGCGGAAGGUCCCUCAGAC) synthesized by Sigma. $100 \mathrm{nM}$ of siRNA duplexes were transfected into cells using Lipofectamine 2000 (Invitrogen) according to the manufacturer's recommendation for 48 hours, following which the expression of FOXM1, NPM and ARF were analyzed by western blotting. Transient transfection with $5 \mu \mathrm{g}$ of PLKO1 or shRNA\#70 from the TRC (Thermo Scientific library) [5] was carried out for the transient NPM knockdown.

\section{Western blotting}

The cells were harvested and lysed following the transient transfection by using IP buffer $(20 \mathrm{mM}$ HEPES, 1\% Triton X-100, $150 \mathrm{mM} \mathrm{NaCl}, 1 \mathrm{mM}$ EDTA, $1 \mathrm{mM}$ EGTA, $100 \mathrm{mM} \mathrm{NaF}, 10 \mathrm{mM} \mathrm{Na} 4 \mathrm{P}_{2} \mathrm{O}_{7}, 1 \mathrm{mM}$ sodium orthovanadate, $0.2 \mathrm{mM}$ PMSF supplemented with protease inhibitor tablet (Roche Applied Sciences)). Protein concentration was determined by the Bio-Rad Protein Assay reagent (BIO-RAD). Isolated proteins were separated on SDS-PAGE and transferred to PVDF membrane (Millipore). Immunoblotting was carried out with antibodies specific for FOXM1, NPM and ARF purchased from Santa Cruz Biotechnology. $\beta$-actin (Sigma) was used as a loading control.

\section{Densitometry}

Densitometry was performed on the scanned immunoblot images using the ImageJ gel analysis tool. The gel analysis tool was used to obtain the absolute intensity (AI) for each experimental band of FOXM1, NPM and ARF followed by transient knockdown of ARF, NPM and FOXM1. The relative intensity (RI) was calculated for each experimental band by normalizing the experimental AI to the cor- responding loading control ( $\beta$ actin).

\section{Results and discussion}

It has previously been shown that FOXM1 interacts with NPM1 [5] and ARF [9] and that ARF interacts with NPM1 [10]. Additionally, it has also been demonstrated that the knockdown of NPM leads to a down-regulation of FOXM1 (Bhat, 2011\} and ARF [11] in various cell types. In the previously published studies we have demonstrated that proteasome inhibitors that usually stabilize majority of the cellular proteins by inhibiting their degradation paradoxically suppress expression of FOXM1, NPM1 and ARF proteins $[12,13]$. We have also shown that proteasome inhibitors to some extent suppressed the mRNA of NPM1 and ARF [13].

In order to evaluate the role of the interaction between FOXM1, NPM and ARF proteins on their stability in Hela cells we used RNA interference. The use of HeLa cells was carried out in this study as it is known to express all three proteins [13]. First, we depleted the cells of FOXM1 using FOXM1 siRNA and evaluated the expression of NPM and ARF. We observed that transient FOXM1 knockdown induced suppression of ARF and NPM protein levels (Fig 1A). In order to validate this observation we repeated this study in three separate experiments. The suppression of NPM and ARF proteins was measured by densitometry. The intensity of the protein bands was normalized to $\beta$-actin intensity. A 70\% inihibition of NPM and almost $90 \%$ inhibition of ARF protein was observed following treatment with 100 nM FOXM1 siRNA for a 48 hour treatment period (Fig 1B). A greater suppression of ARF as compared to NPM1 could be explained by the fact that NPM is abundantly expressed in most cancer cells. The qualitative and quantitative measurement validated the observation that suppression of FOXM1 by siRNA leads to downregulation of NPM1 and ARF proteins.

Next the effect of NPM1 suppression on FOXM1 and ARF protein levels was observed. We found that the transient knockdown of NPM1 by shRNA in HeLa cells for 48 hours led to the suppression of FOXM1 and ARF proteins (Fig 2A) as has been shown in different cell types previously $[5,11]$. We also measured the percent of supresssion of FOXM1 and ARF proteins by densitometric analysis. We found that transient knockdown of NPM1 led to inhibtion of up to $80 \%$ of ARF and FOXM1 expression as compared to control (PLKO1) transfected cells (Figure 2B).

Finally we decided to examine how the suppression of ARF by RNA interference could affect FOXM1 and NPM1 protein expression. The HeLa cells were transiently transfected with $100 \mathrm{nM}$ ARF siRNA 
for 48 hours following which the expression of FOXM1 and NPM proteins were investigated. We observed that a transient inhibition by ARF-siRNA led to the inhibition of NPM and FOXM1 proteins (Fig $3 \mathrm{~A})$. The suppression was further validated in three separate experiments. The quantitative measurement of suppression was performed by densitometry. It was seen that suppression of ARF by RNAi inhibited up to $65 \%$ of NPM and up to $85 \%$ of FOXM1 expression (Fig 3B). Interestingly, we found that a tumor suppressor protein ARF stabilizes two proteins with oncogenic properties, NPM1 and FOXM1. The biological significance of this phenomenon needs to be investigated. Our data suggest that, in cancer cells, depletion of any one of FOXM1/NPM1/ARF protein leads to down-regulation of the two other proteins.
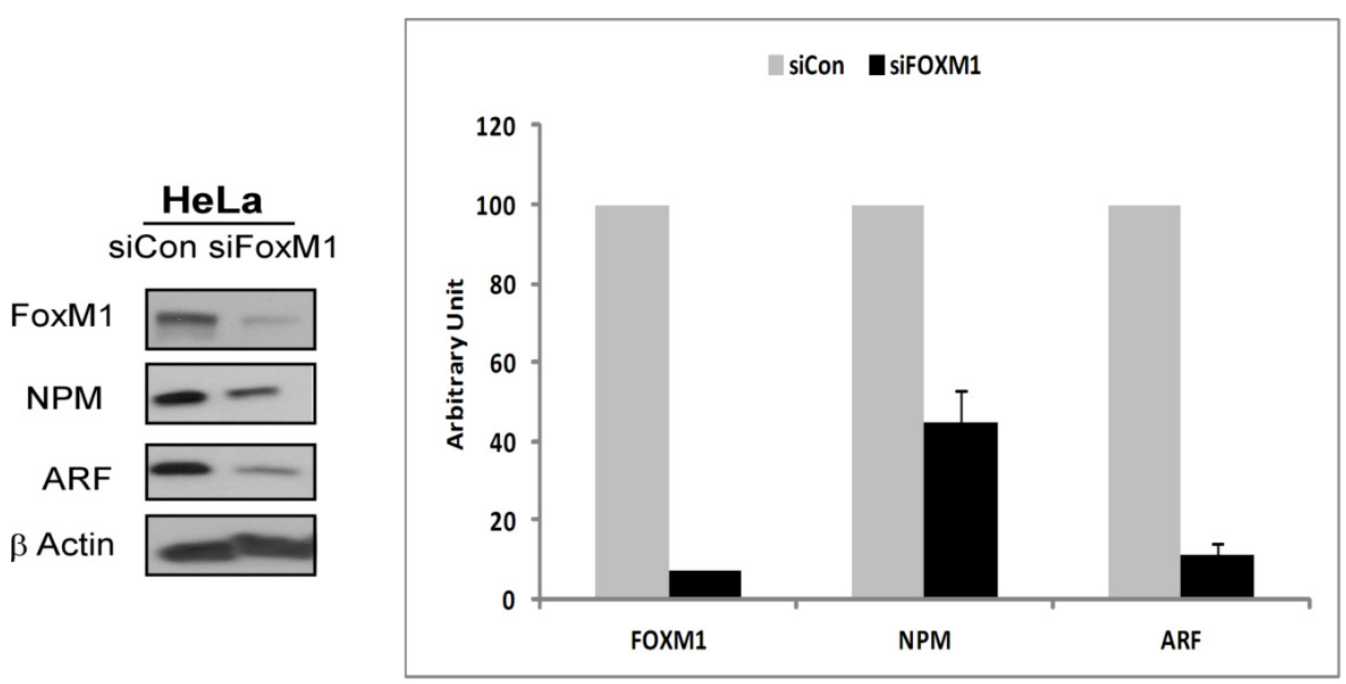

A.

B.

Figure 1. Suppression of FOXMI by RNA interference in HeLa cells leads to downregulation of NPM and ARF proteins. A. HeLa cells were transiently transfected with a control small interfering RNA (siRNA) and siRNA specific to FOXMI according to the manufacturer's recommendation for 48 hours, following which the expression of FOXMI, NPM and ARF were analyzed by western blotting. $\beta$-actin (Sigma) was used as a loading control. FOXMI, NPM and ARF antibodies were purchased from Santa Cruz Biotecnhnology. B. The band intensities of FOXMI, ARF and NPM proteins were quantified by densitometry using the Image J gel analysis tool. Columns, mean of three experiments; bars, SD;

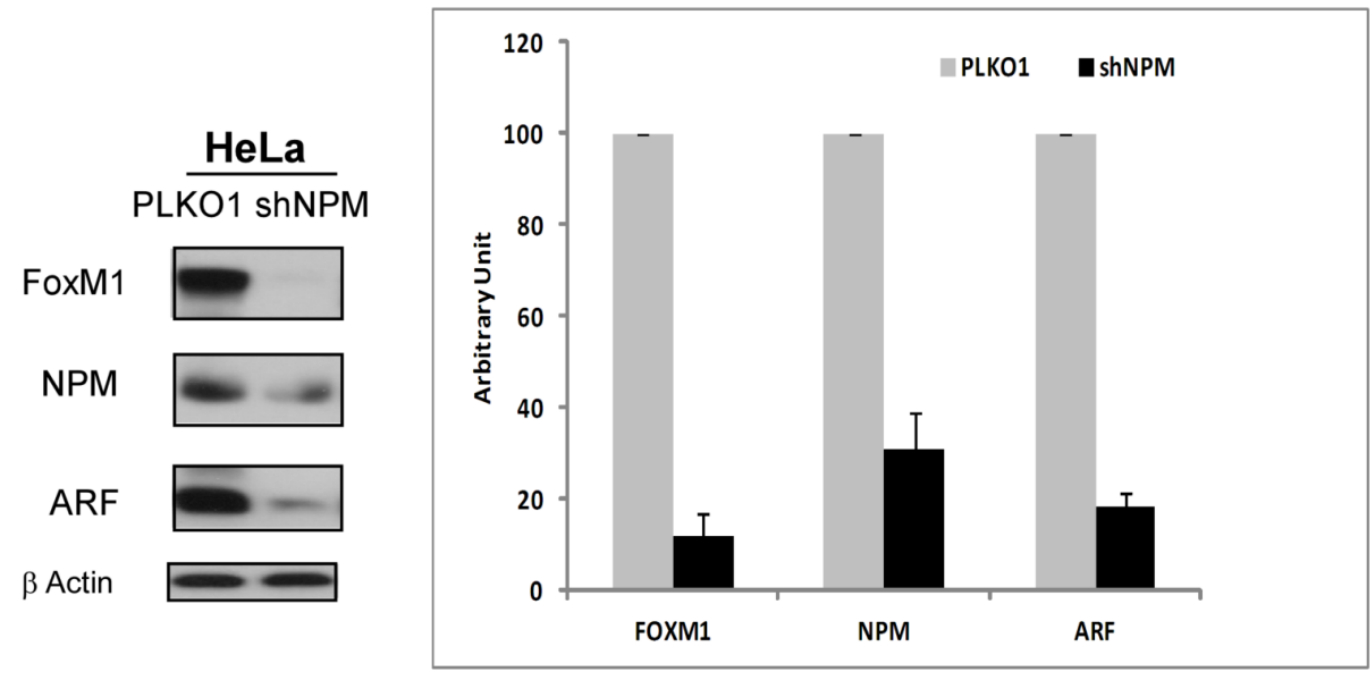

A

B

Figure 2 Suppression of NPM by shRNA in HeLa cells leads to downregulation of ARF and FOXM1 proteins. A. The protein levels of FOXMI, ARF and NPM following transient NPM knockdown by lentiviral transduction in HeLa human cervical cancer cell line were determined by immunoblotting. $\beta$-Actin was used as the loading control. B. The band intensities of FOXMI, ARF and NPM proteins were quantified by densitometry using the Image J gel analysis tool. Columns, mean of three experiments; bars, SD; 


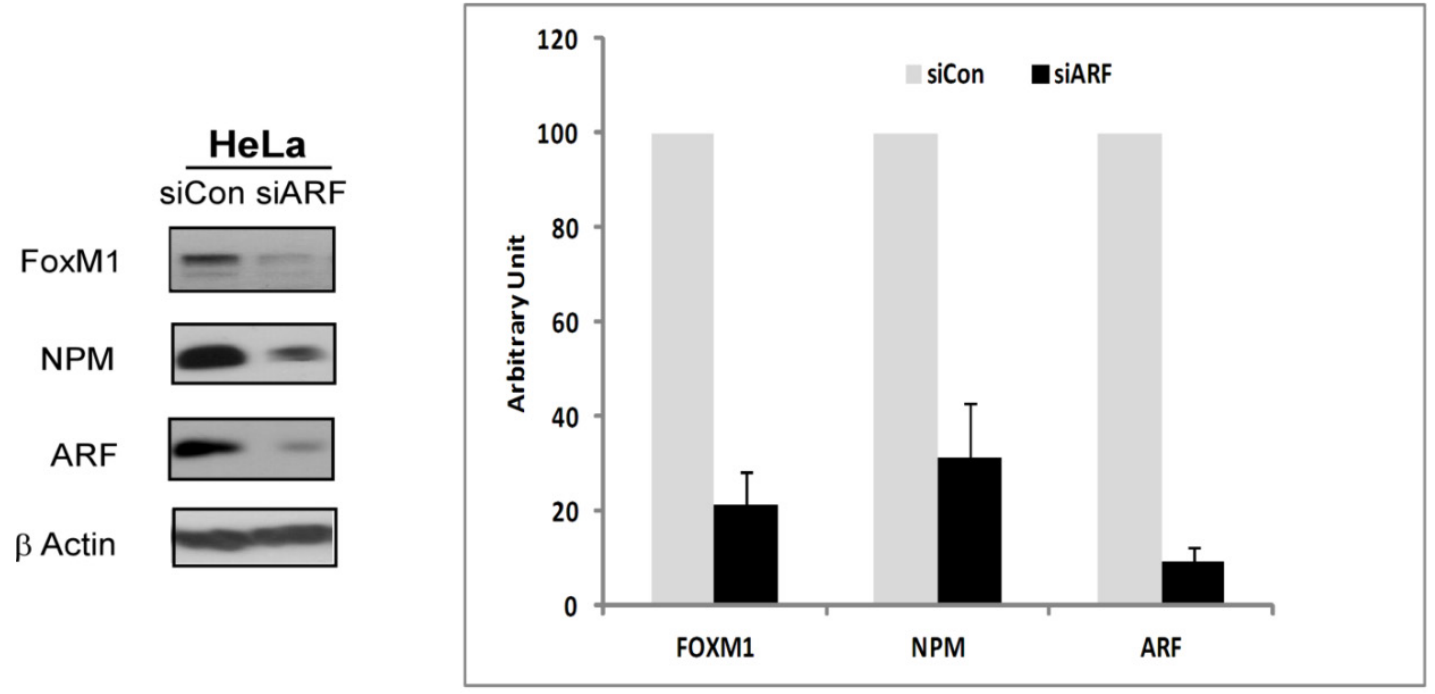

A.

B.

Figure 3. Suppression of ARF by RNA interference in HeLa cells leads to downregulation of NPM and FOXM1 proteins. A HeLa cells were transiently transfected with a control siRNA or ARF-siRNA following which the expression of FOXMI, NPM and ARF were analyzed by immunoblotting. $\beta$-actin was used as a loading control. B. The band intensities of FOXMI, ARF and NPM proteins were quantified by densitometry using the Image J gel analysis tool. Columns, mean of three experiments; bars, SD.

\section{Acknowledgments}

This work was supported by NIH grants 1RO1CA129414, RO1CA138409, UIC Inception Grant and 2014 Cancer Center pilot project to ALG.

\section{Competing Interests}

The authors have declared that no competing interest exists.

\section{References}

1. Halasi M, Gartel AL: FOX(M1) News--It Is Cancer. Molecular cancer therapeutics 2013, 12(3):245-254.

2. Pilarsky C, Wenzig M, Specht T, et al.: Identification and validation of commonly overexpressed genes in solid tumors by comparison of microarray data. Neoplasia (New York, NY 2004, 6(6):744-750.

3. Halasi M, Gartel AL: Targeting FOXM1 in cancer. Biochemical pharmacology 2013, 85(5):644-652.
4. Brady SN, Maggi LB, Jr., Winkeler CL, et al.: Nucleophosmin protein expression level, but not threonine 198 phosphorylation, is essential in growth and proliferation. Oncogene 2009, 28(36):3209-3220.

5. Bhat UG, Jagadeeswaran R, Halasi M, Gartel AL: Nucleophosmin interacts with FOXM1 and modulates the level and localization of FOXM1 in human cancer cells. The Journal of biological chemistry 2011, 286(48):41425-41433.

6. Grisendi S, Mecucci C, Falini B, Pandolfi PP: Nucleophosmin and cancer. Nat Rev Cancer 2006, 6(7):493-505.

7. Sherr CJ: Divorcing ARF and p53: an unsettled case. Nat Rev Cancer 2006, 6(9):663-673.

8. Ozenne P, Eymin B, Brambilla E, Gazzeri S: The ARF tumor suppressor: structure, functions and status in cancer. International journal of cancer 2010, 127(10):2239-2247.

9. Kalinichenko VV, Major ML, Wang X, et al. Foxm1b transcription factor is essential for development of hepatocellular carcinomas and is negatively regulated by the p19ARF tumor suppressor. Genes $\mathcal{E}$ development 2004, 18(7):830-850.

10. Korgaonkar C, Hagen J, Tompkins V, et al. Nucleophosmin (B23) targets ARF to nucleoli and inhibits its function. Molecular and cellular biology 2005, 25(4):1258-1271.

11. Lo D, Zhang Y, Dai MS, et al. Nucleostemin stabilizes ARF by inhibiting the ubiquitin ligase ULF. Oncogene 2014.

12. Bhat UG, Halasi M, Gartel AL: FoxM1 is a general target for proteasome inhibitors. PLoS ONE 2009, 4(8):e6593.

13. Pandit B, Gartel AL: Proteasome inhibitors suppress expression of NPM and ARF proteins. Cell cycle 2011, 10(22):3827-3829. 